\title{
What motivates social workers to become practice teachers?
}

\section{Deborah Develin $^{1}$ and Ian Mathews ${ }^{2}$}

Summary: The introduction of the new social work award in September 2003 placed a greater emphasis on students learning from practice and as a consequence of this, the demand for assessed practice learning opportunities increased. This paper will outline research undertaken in response to the challenges that this has created by examining the central question of what factors motivate social worker practitioners to become practice assessors and how influential organisational factors are in individuals choosing to take on the role. The aim of the research was to identify extrinsic and intrinsic motivating factors that might then provide guidance to those involved in recruiting individuals to take on the crucial role of contributing to the education of the next generation of social workers. The findings, using data from 50 self administered questionnaires and two focus group interviews, collected from participants attending introductory practice assessor training indicates that intrinsic factors are principle sources of motivation rather than extrinsic, organisational factors.

Keywords: motivation; practice assessor; continuing professional development; learning organisation; social work education and training; practice learning opportunities

1. Project Manager, Skill for Care, East Midlands

2. Senior Lecturer in Social Work, University of Lincoln

\section{Address for Correspondence:}

Deborah Develin, Skills for Care, Marlborough House, Charnwood Street, Derby DE1 2GT. England. deborah.develin@skillsforcare.org.uk

Ian Mathews, University of Lincoln,, Brayford Pool, Lincoln LN6 7TS. England. imathews@lincoln.ac.uk

\section{Acknowledgements}

To Mark Doel for his advice and guidance, and to all those who assisted with and participated in the research

18 Journal of Practice Teaching \& Learning 8(1) 2008, pp.18-30. DOI: 10.1921/19662. @ w\&b 


\section{Background}

The new social work award, introduced in September 2003, brought about a radical transformation of social work education and training, including the requirement that students complete a minimum of 200 days of practice learning (Department of Health, 2002). The increased emphasis on students learning from practice, coupled with government targets doubling the number of social work students, has brought challenges to those involved in delivering and supporting the social work degree to ensure that there is sufficient quality practice learning opportunities to meet demand. The significant increase in practice learning days has led to an increased demand for practice assessors ${ }^{1}$ and therefore activity to recruit practice assessors has increased, supported by two Department of Health initiatives, the Practice Learning Taskforce and the Learning Resource Network.

\section{Defining motivation}

Motivation, and what motivates workers to undertake tasks, is difficult to define. Implicitly building on the work of such early theorists as Maslow (1943) and his hierarchy of human needs, the majority of studies into motivation have been undertaken in the world of business and commerce where job satisfaction, retention and productivity are key areas of concern. Typically, these studies have emphasised the importance of 'extrinsic' rewards, such as additional pay, fringe benefits, and material goods, and 'intrinsic' rewards, such as personal growth and development (Mullins, 2002)

Mitchell (1982) identified some common characteristics which underlie the definition of motivation. Significantly, he acknowledges that motivation is a unique phenomenon with each individual responding to different prompts. He also notes the multi-faceted nature of motivation; the differences between those factors which initially motivate behaviour and those which sustain, or direct, the ongoing task.

Other theorists have argued that what motivates workers is the content of their work - the intrinsic challenge, and interest of the task itself - while those factors which de-motivate workers are most likely to be associated with the context of work - working conditions, company policy and aspects of supervision (Herzberg, 1959)

Given these uncertainties, it seems wise to adopt an accessible definition

19 Journal of Practice Teaching \& Learning 8(1) 2008, pp.18-30. DOI: 10.1921/19662. @ wEb 
of motivation. Cole (1995) suggests that

motivation is the term used to describe those processes, both instinctive and rational, by which people seek to satisfy the basic drives, perceived needs and personal goals, which trigger human behaviour.

\section{Previous research}

There is very limited research into what motivates social workers to become, and remain, practice assessors. One consistent theme is the role of the employing organisation in stimulating and supporting individual workers to become practice assessors. Organisations which have a culture of encouraging placements, providing workload relief, releasing staff to attend training and briefing sessions, providing office space for students, and providing administrative support, tend to generate and keep practice assessors (Globerman \& Bogo, 2003; Rosenfeld, 1989; Doel, 2006). Support from universities in providing quality training for practice assessors, providing visits to students on placement, and support with concerns also motivated staff to become and remain practice assessors (Rosenfeld, 1989; Bogo and Power, 1992).

Personal growth and development is also consistently highlighted. The process of teaching students from outside the organisation stimulated critical thinking and reflective practice, leading to an enhanced sense of professionalism and currency (Globerman \& Bogo, 2003; Lindsay \& Thompsett, 1998). This emphasis on professional and personal development, especially the opportunity to update and enhance skills and knowledge, has been highlighted as a motivational factor in why social workers undertake post qualifying study (Doel, et al, 2008). Given the correlation between practice assessor work and the post qualifying framework this is hardly surprising. An increase in status or organisational importance is not a factor (Kahn, 1981; Lacerte et al, 1989), although a feeling of 'a job well done' and satisfaction with the task was significant (Bogo \& Power, 1992). It is also recognised that students tend to 'freshen up' an organisation and challenge established ways of working and the professional practice of their assessors. Interestingly, Globerman and Bogo (2003) note that this rarely extends to challenging the values of the individual practice assessor, but is situated at a more organisational level.

Finally, some practice assessors undertake the task because they want

20 Journal of Practice Teaching \& Learning 8(1) 2008, pp.18-30. DOI: 10.1921/19662. @ wE-b 
to give their expertise and knowledge back to the profession. A significant proportion of practice assessors are experienced professionals who have worked in social care for many years. Whilst this motivation is essentially altruistic, Globerman and Bogo (2003) note that it is tinged with a desire to imprint the assessors standards, professionalism, and understanding of the role onto the student.

\section{What motivates social workers to become practice assessors?}

\section{Research method}

The research process entailed collecting qualitative and quantitative data, through self administered questionnaires and focus group interviews about factors that motivate social workers to become practice assessors.

After piloting, minor amendments were made to the questionnaire before it was distributed to all participants attending introductory five day practice assessor training programmes over a six month period.

The questionnaire consisted of two open questions and thirteen scaled closed questions. Data gathered from closed questions was analysed using the mode and open questions were coded and analysed. The method of distribution resulted in a high response rate of $96 \%$ : out of 52 potential responses 50 questionnaires were returned.

Key themes emerging from the questionnaire analysis were followed up in two focus groups. Participants were identified from questionnaire respondents indicating a willingness to take part. Due to practical difficulties, namely the geographical size of the area of study and work pressures of potential participants numbers in each group was low.

Respondents were given the opportunity at the focus groups to provide open, non-directed responses to the main research question. Following this, the data was coded and analysed.

The questionnaires gave a very clear picture of why staff, busy and over stretched as they were, chose to undertake the role of practice assessor (see Table 1). The questionnaires indicated that

- $78 \%$ of respondents stated that to have an opportunity to contribute

21 Journal of Practice Teaching \& Learning 8(1) 2008, pp.18-30. DOI: 10.1921/19662. @ wEb 


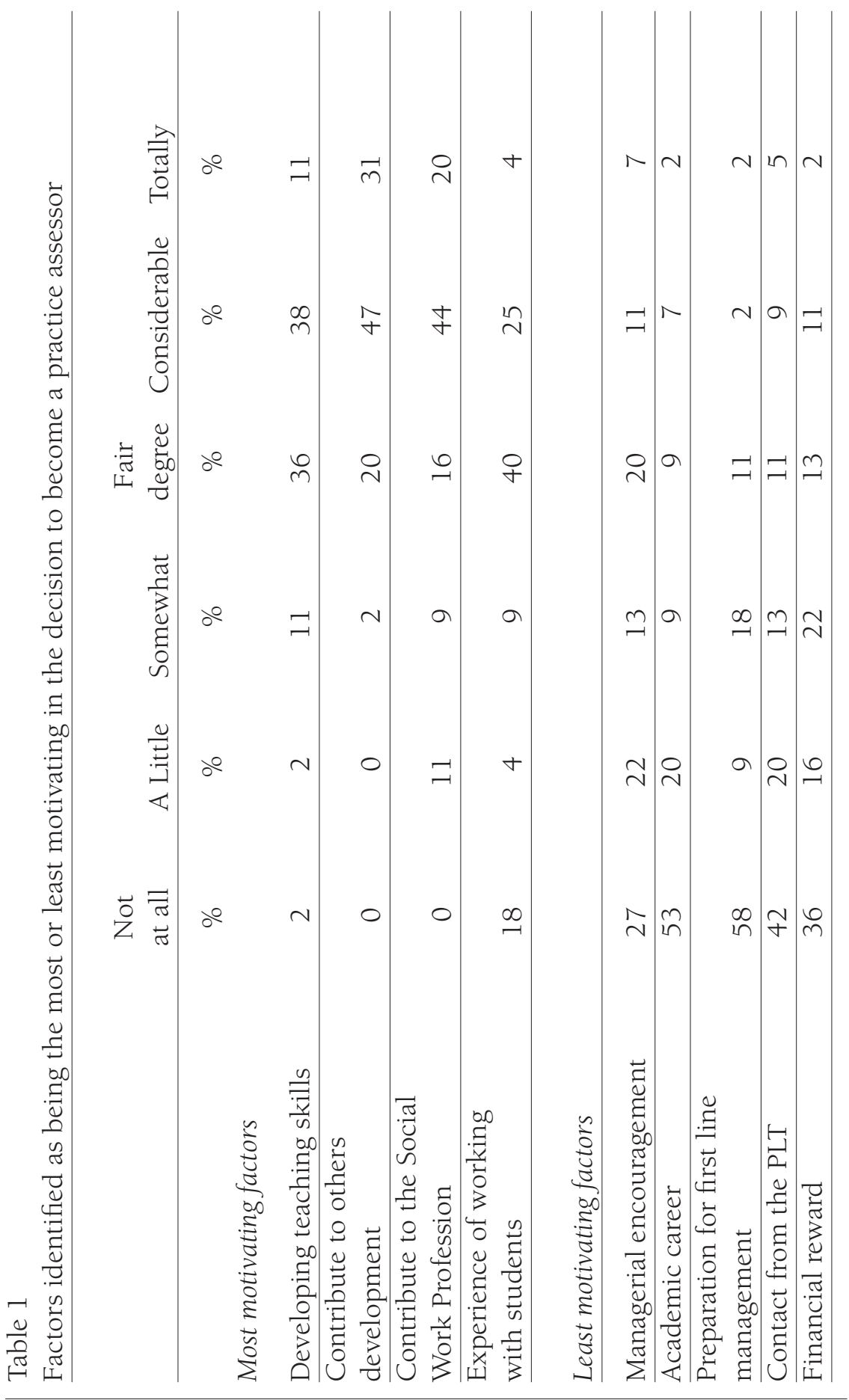

22 Journal of Practice Teaching E Learning 8(1) 2008, pp.18-30. DOI: 10.1921/19662. (C) w Eb 
to the development of others was 'considerably' or 'totally' significant to their decision

- $64 \%$ of respondents stated that the desire to make a contribution to the social work profession was 'considerably' or 'totally' significant to their decision

These were the most popular reasons given and reinforce the notion of altruism, and the desire to 'give something back', that underpins the ethos of public service. Social workers are often seen as being public spirited, conscience led, and as having a 'generosity of spirit' - attributes reinforced during the General Social Care Council's 2007 enquiry into the definition of the roles and tasks of social work (GSCC, 2007)

The focus groups explored what respondents were referring to in these responses and found that the underlying reasons were not as altruistic as they may seem. For example, there seemed to be an emphasis on students learning how to practice in exactly the same way as the practice assessor, on the basis that this was good practice and should be emulated. There was little recognition of difference, or an appreciation that students could gain from other colleagues or different styles of work.

There was also a motivation to influence the student away from what some saw as a computer led, functional approach to social work and back to a more value-based, service user perspective. For example, from a focus group member who reminisced that

Practice has changed ... there is less interaction with people and more interaction with the computer ... practice assessors can demonstrate what is important and challenge students not to loose sight of children and their families.

It was implicitly assumed that this process driven approach was inculcated into students by their social work education. Whilst the divide between contemporary social work education and the training received by many of the respondents in the 1990s might not be as wide as assumed, there was almost a discernible clash between what was perceived as traditional social work, and a new more mechanistic form of practice. This is suggestive of disenchantment by the practice assessor with the pressures of practice, and the increasing emphasis on outcomes and processes, as opposed to any thoughtful criticism of social work education.

Another significant motivational factor was to 'give something back'

23 Journal of Practice Teaching \& Learning 8(1) 2008, pp.18-30. DOI: 10.1921/19662. C wEb 
to the social work profession. Respondents were strongly committed to social work, as evidenced by their desire to become practice assessors, and spoke positively of the life chances and rewards that they had received from the profession; although this seemed to be in contradiction to the disillusionment mentioned previously. In terms of what it was that they were 'giving back' respondents essentially wanted to transmit, or pass on, the values that they saw as being integral to the profession. This corresponded with the desire to mould students into workers who were driven by traditional social work values. There was, however, less explicit agreement about what those values were. Implicitly, respondents alluded to such values as choice, empowerment, and respect, but above all seemed to focus their thoughts on the uncertain notion of service user centrality.

The third most popular motivational factor was the opportunity to develop teaching skills, which contributed either 'considerably' or 'totally' to the decision making of $49 \%$ of the cohort. This was interesting as it was unclear why practice assessors wanted to develop these skills. One notable feature of the research is that very few respondents had any desire to enter an academic career - only $2 \%$ indicated that this was the total motivating factor in their decision. A further theme to emerge was a belief that these transferable skills would be helpful in other areas of work, and for others it was the mental challenge and stimulation of developing a different skill.

One focus group member felt that in the competitive world of social work employment, it was important to have as many skills and qualifications as possible to offer a potential employer.

He suggested that being a practice assessor made him

more marketable in an uncertain and changing social care world where unqualified staff are increasingly taking on roles traditionally done by social workers. If you have more strings to your bow then you are more likely to be successful at interview.

Given the increasing fragmentation of social work and the threat posed by cut backs and reorganisations, this view may have even greater credence in future years.

The fourth most popular factor, was the experience staff had had of working with students with 64\% saying this had influenced their decision a 'fair degree' or 'considerably'. These were not necessarily

24 Journal of Practice Teaching E Learning 8(1) 2008, pp.18-30. DOI: 10.1921/19662. (๐ wËb 
social work degree students and included NVQ, occupational therapy and nursing students. Whilst they had not had prime responsibility for their learning, it was clear that having students in their teams had been a positive experience. Staff seemed to enjoy the energy and enthusiasm that they brought and hoped to repeat the experience with social work students. It is perhaps of significance that no one had experience of a failing student. This is echoed in research by Parker and Whitfield (2006) examining the link between practice learning and recruitment and retention where almost half of survey respondents saw practice learning as a team activity, which suggests the development of a learning culture in some teams.

The focus groups also identified that respondents had had diverse experiences whilst on placement themselves, and that this was influential in motivating them.

For example, one respondent stated that she had a

poor experience as a student - the practice assessor misused their power and received no support from the university.

Another respondent had a similarly poor experience

I didn't have a rigorously assessed placement, and I was aware of the power difference in our relationship.

Both respondents were motivated to 'do better' as practice assessors themselves and to create a better climate for learning.

Conversely, other respondents felt that they had had a very good placement themselves and had a positive relationship with their practice assessor. This experience equally acted as a motivating factor, with the respondent implicitly suggesting that they were going to model their practice on their practice assessor.

Organisational influences were equally contradictory. It was disappointing that many respondents reported that their organisations were ambivalent towards practice assessment and had not made any link with benefits such as recruitment and retention, or the meeting of performance indicators.

For example, one respondent stated that there was

no organisational pressure to take students - my work place is unaware that

25 Journal of Practice Teaching \& Learning 8(1) 2008, pp.18-30. DOI: 10.1921/19662. @ wEb 
practice learning is a key performance indicator.

More worryingly still, one focus group member said that

the treatment of practice assessors by the organisation puts people off ... you only do it if it benefits you personally rather than the organisation.

The respondent was referring to a general lack of recognition, support or case load relief in her remarks. Such hostility to the task was not openly voiced by others, but it raises the question of how many staff have been actively dissuaded from practice assessing due to a lack of interest, and organisational ambivalence.

Conversely, another respondent found that her organisation had made a clear link between practice assessment and recruitment, and actively encouraged her to go forward for training.

Recruitment to the organisation in the future is a driver within the organisation to take students - the question has been asked, where will the future workforce come from? There is an increasing recognition within the organisation of the link between student placements and recruitment.

She further reported that historically her team had not taken students due to the perceived need for them to have a certain level of experience. This view had changed over the last twelve months as it became clear that recruitment was becoming more difficult.

\section{What does not motivate social workers to become practice assessors?}

As the research gave some clear indications as to why practitioners were motivated to undertake the task of practice assessor, it was also helpful in highlighting reasons which did not motivate staff (see table 1)

It is an often stated piece of 'practice wisdom' that practice assessing, with its emphasis on supervision, management and learning, is a helpful first step into either management or academia. Whilst this may be true, there was no evidence to suggest that respondents chose to undertake the task for these reasons. On the contrary, 58\% of questionnaire

26 Journal of Practice Teaching \& Learning 8(1) 2008, pp.18-30. DOI: 10.1921/19662. @ w\&b 
respondents stated that 'preparing for a job in management' was 'not at all' a factor in their decision making. This was mirrored by a further 53\% who stated that 'preparation for an academic career' was 'not at all' a factor in their decision making. The latter was further clarified by the very low number of respondents who saw practice assessing as a means of strengthening their connections with local universities.

The overall tenor of the research is that practice assessing is undertaken for individual reasons connected to challenge, altruism and stimulation, rather than any well planned attempt to launch a career. This was further reinforced by focus group respondents who openly stated that they had 'no interest in management' and 'had not thought of a career in academia.' It could be argued that it is this disinterest and the lack of career opportunities for practitioners outside of management that motivates practitioners to become practice assessors. In other words, it is precisely because practitioners do not want a career in management or academia which motivates them to become practice assessors.

Over recent years, various attempts have been made to attract practitioners to the role of practice assessor. Local Authorities have spent considerable amounts of money on developing dedicated practice learning teams, and on offering financial inducements to staff. Neither of these was of significance in attracting staff to the role. Only $2 \%$ of questionnaire respondents indicated that financial reward was foremost in their minds, whilst 36\% stated that it was not at all important. This is supported by Doel's research (2006) into factors for successful practice learning in Local Authorities.

Equally, direct contact from practice learning team staff did not appear to be effective with over $60 \%$ of questionnaire respondents stating that no contact had taken place or if it had, it had not been a factor. A number of focus group respondents indicated that they were not aware that their organisations had practice learning teams until training had been organised.

At first sight, managers also appear to have had little influence in motivating staff. What the focus groups revealed, however, was that a small number of managers were very instrumental in encouraging their staff to consider practice assessing, essentially as a means of furthering their professional development. Where managers were pro-active they seem to have had a major influence, although many questionnaire respondents reported that their manager had not encouraged them at all. It did not seem to make any difference whether the manager themselves

27 Journal of Practice Teaching \& Learning 8(1) 2008, pp.18-30. DOI: 10.1921/19662. @ wEb 
had been a practice assessor. Whilst there were not adverse comments about the content or regularity of line management supervision, it is perhaps indicative of the fact that social work supervision is traditionally not good at encouraging staff to develop and 'move on.' The importance of managerial encouragement and support in practitioners deciding to become practice assessors was highlighted by Parker and Whitfield (2006) in their research into the link between practice learning and recruitment and retention. It is also concerning that very few respondents had made the link between the activity of practice teaching and maintaining professional registration, or saw practice assessing as a means of gaining the PQSW. Whilst it would not be wise to speculate on the reasons for this, it demonstrates a lack of awareness and an inability to see practice teaching within a wider context of continuing professional development.

\section{Conclusion}

The objective of the research was to understand the factors that motivate social work practitioners to take on the role of practice assessor. Key themes and patterns have been highlighted, a limitation of the study is the lack of generalisability, however the findings have implications for those involved in recruiting practice assessors.

Unsurprisingly, this research has demonstrated that social workers come to practice assessing for a range of diverse reasons, primarily individual rather than organisational. The principal reasons seem to be a complicated amalgam of a willingness to contribute to the development of others, and the need to find personal satisfaction and stimulation. Prompting from external sources did not seem to be particularly influential, although, significantly, the actual experience of having students around in the team seemed to make a difference and was recognised as good practice. Organisations need to make better use of the willingness to engage with students and find ways of formally encouraging staff to consider practice assessing. Ensuring practice learning is embedded within the strategic thinking of organisations is a means of achieving this. Developing a structure where practice assessing is included in job roles and is part of practitioners ongoing continuing professional development needs will help to develop a learning culture,

28 Journal of Practice Teaching E Learning 8(1) 2008, pp.18-30. DOI: 10.1921/19662. (๐ wËb 
benefiting students, practitioners and the organisation.

Motivation and motivating factors are not easy to tease out, and further work is required, particularly around the issue of what continues to motivate practice assessors who undertake the task for many years.

\section{Editor's note}

1. The terms 'Practice Assessor' and 'Practice Teacher' are now used interchangeably in England and relate to a role that focuses on assessing, teaching, supervising and managing the learning of a social work student who is undertaking a practice learning placement.

\section{References}

Bogo, M. and Power, R. (1992) New field instructors' perceptions of institutional supports for their roles., Journal of Social Work Education, 35, 265-274

Cole, G. (1995) Organisational behaviour. Continuum,

Department of Health (2002) Requirements for Social Work Training. London: $\mathrm{DoH}$

Doel, M. (2006) Effective Practice Learning in Local Authorities. 1. Strategies for improvement. (Practice Learning Taskforce Capturing the Learning series) London: Practice Learning Taskforce

Parker, J. and Whitfield, J. (2006) Effective Practice Learning in Local Authorities. 2. Workforce development, recruitment and retention. (Practice Learning Taskforce Capturing the Learning series) London: Practice Learning Taskforce

Doel, M., Flynn, E., and Nelson, P. (2008) Experiences of post-qualifying study in social work. Social Work Education, 27, 5, 549-571

General Social Care Council (2007) 'Describing social work roles and tasks: A record of the work that took place at the stakeholder event in London, 14th February, 2007' www.gscc.org.uk

Globerman, G. and Bogo, M. (2003) Changing times: Understanding social workers' motivation to be field instructors', Social Work, 48, 1, 65-73

Herzberg, F. (1959) The Motivation to Work. (2nd ed.) Chichester: John Wiley Kahn, S. (1981) An Analysis of the Relationship between Social Work Schools and Field Placement Agencies in Their Joint Tasks of Educating Social Workers.

29 Journal of Practice Teaching \& Learning 8(1) 2008, pp.18-30. DOI: 10.1921/19662. @ wEb 
(unpublished dissertation) New York: Columbia University

Lacerte, J; Ray, J.; and Irwin, L. (1989) Recognising the educational contributions of field instructors. Journal of Teaching in Social Work, 3, 99-113

Lindsay, J. and Tompsett, H. (1998) Careers of Practice Teachers in the London and South East Region. London: CCETSW

Maslow, A. H. (1943) 'A theory of human motivation. Psychological Review, 50, 370-396

Mitchell, T.R. (1982) Motivation: New directions for theory, research and practice. Academy of Management Review, 7th January

Mullins, LJ. (2002) Management and Organisational Behaviour. (6th ed.) London: Prentice Hall

Rosenfeld, D.J. (1989) Field instructor turnover. in M. Raskin (Ed.) Empirical Studies in Field Instruction. Binghampton, NY: Haworth press (pp. 187218) 